\title{
Blood pattern analysis-a review and new findings
}

Prashant Singh ${ }^{1 *}$, Nandini Gupta ${ }^{1}$ and Ravi Rathi ${ }^{2}$

\begin{abstract}
Background: Blood is one of the most common pieces of evidence encountered at the crime scene. Due to the viscous nature of blood, unique bloodstain patterns are formed which when studied can reveal what might have happened at the scene of the crime. Blood pattern analysis (BPA), i.e., the study of shape, size, and nature of bloodstain. The focus of this paper is to understand blood and BPA. An experimental finding to understand blood stain formation using Awlata dye was conducted within the university premises under laboratory conditions. Awlata (Alta), an Indian dye used for grooming of women, was used to create fake blood stains to understand the formation of bloodstains with respect to varying heights, and their relation with spines and satellite stains was determined.

Results: When the height of dropping fake blood increased, the distance of satellite stains emerging from the fake blood stains was also increasing. From the experimental finding, it was found that satellite stains were directly proportional to height of blood stain and spines were inversely proportional.

Conclusion: It can be concluded that blood is a vital source of information and when interpreted correctly it can be used as a source of information that can aid in investigations. Thus, a relation between formation of blood stains with relation to height was established. This finding using fake blood stains can help in carrying out future studies.
\end{abstract}

Keywords: Awlata, Blood, Bloodstains, Crime, Evidence

\section{Background}

The study aims to determine the relationship between spines and satellites stains in accordance with varying heights using Awlata dye. It involves creation of fake bloodstains using Awlata dye to determine this relation. The study also seeks to suggest the use of Awlata dye for studying bloodstains for conducting future studies.

Blood is an organic fluid circulating in our body that is essential to maintain life; it includes blood cells and plasma that accounts for approximately $8 \%$ of body weight. Blood ranges from 4-5 L (female) to 5-6 L (male). Blood has few bodily capabilities which can be required for its morphological interpretation like specific weight, viscosity, and surface tension (Peschel et al. 2011; Bevel

\footnotetext{
* Correspondence: singhp1103@gmail.com

${ }^{1}$ School of Forensic Science, National Forensic Sciences University, Gandhinagar, Gujarat, India

Full list of author information is available at the end of the article
}

and Gardner 2012). Viscosity in terms of blood may be described as the pressure of the flow of blood, due to shear stress or extensional stress inside the body (Bevel and Gardner 2012). An elastic-like property of a fluid due to cohesive forces between liquid molecules is surface tension (Larkin et al. 2012). Blood possesses fluid nature inside the body or when it exits from the body due to an impact/injury (James et al. 2005). If there were blood clots in the blood found at the crime scene, it suggests that the victim was exposed to an extended injury (Peschel et al. 2011; Bevel and Gardner 2012).

Blood can exit from the body as drip, spurt, etc., or can even ooze from wounds depending on the type of infliction/damage. BPA is a type of examination that includes the interpretation of shapes of the bloodstains (James et al. 2005). Blood pattern analysis aims to reveal the physical events that might have occurred at the crime scene. These bloodstains can be interpreted by 
their shape, size, and distribution (Brodbeck 2012). The facts acquired from BPA can help in crime scene reconstruction, corroborating witness statements, for the investigative procedure (James et al. 2005). If bloodstains at a crime scene are either dried or removed by the assailant, they can still be recovered by spraying luminol. Luminol (5-amino-2,3 dihydro-1,4-pthalazine-dione) can be used to detect the presence of minor, unnoticed, or hidden bloodstains diluted down to a level of $1: 10^{6}(1 \mu \mathrm{L}$ of blood in $1 \mathrm{~L}$ of solution) which gives chemiluminescence or glowing effect when it reacts with dried bloodstains (Quickenden and Creamer 2001).

Luminol solution is usually directly sprayed in completely dark environments, and then UV (ultra violet) light visualizes the sample (blood). The fluorescence obtained is then photographed or filmed. Luminol can be used to identify minor, unnoticed, or hidden bloodstains, and it also has a high sensitivity to old blood or completely dried blood but, unfortunately, luminol can react with detergents, metals, and vegetables to give falsepositive results (Barni et al. 2007). Sometimes, there are probabilities that the bloodstain recovered had been created using certain substances (dyes/stains) to deceive the investigators. To distinguish whether a sample is blood or not, assays like Kastle-Meyer (phenolphthalein test), Medinger reaction (Leuco malachite Green), and Tetramethylbenzidine test are used, but they cannot satisfactorily confirm blood (preliminary tests). So, for the confirmatory evaluation of blood, Teichmann and Takayama tests are performed to distinguish if the samples were blood or not (Saferstein and Hall 2020). It is also very important for the analyst to determine the origin of species of blood (whether human or animal) by precipitin test; this is often necessary to avoid confusion in investigative findings. There are several conditions in which the bloodstain patterns are disturbed/altered and in such cases, no useful information can be interpreted. So, DNA analysis is utilized for providing investigative leads (Saferstein and Hall 2020). When the bloodstains are suspected to be from multiple sources, the investigator can often rely on DNA to reveal valuable details about the crime. So, in the case of multiple victims, analysts often use DNA profiling to determine whose blood it was (James et al. 2005; Karger et al. 2008).

Bloodstain patterns distributed at the crime scene can be used for the reconstruction of an event (Comiskey et al. 2016). Before reconstruction, an analyst must have a comprehensive view of the overall picture and use the step-by-step approach to differentiate and analyze the bloodstain patterns and search for the informative points (James et al. 2005). It is also required that the investigator must create a hypothesis on the formation of blood patterns due to injuries. Reconstruction can be further improved by the contribution of case descriptions and statements (witnesses/perpetrators) that can provide insights on the sequence of events. Hence, to carry out an effective reconstruction, both casework experience combined with knowledge of injuries should be known (Karger et al. 2008; Kunz et al. 2013; Kunz et al. 2015).

\section{Types of bloodstains \\ Passive patterns}

It is a type of bloodstain pattern formed due to gravity, patterns like drip stain, flow stain, blood pool, and serum stain are observed. A drip stain is a drop falling without any disturbance that can take a spherical shape without disintegrating into smaller droplets. Bloodstains, depending on the angle, can cause the blood drop to have a circular or slightly elongated shape; this helps in the determination of the angle of impact (Swgstain 2009). Sometimes, a trail can be formed due to the dripping of blood from a weapon as well as in case of blunt or trauma injuries, due to which large volume of blood can be encountered at the crime scene (James et al. 2005; Peschel et al. 2011).

\section{Spatter patterns}

These are patterns formed when hard objects are used to strike the victim (example: a pipe). Forward spatter on the other hand is a pattern formed towards the direction of damage (example: bullet creating an exit wound) (James et al. 2005; Peschel et al. 2011). Back spatter is a pattern formed by blood when damage is to a hard surface like the skull by a bullet, and the bloodstains will be pointing away from the impact. Gunfire spatter can also vary on the caliber of the weapon used, location of impact, and the location of the victim (James et al. 2005; Peschel et al. 2011).

Projected patterns are irregular patterns that are due to the motion of weapon (example: stabbing). If in case at the crime scene there was existence of droplets of blood of varied sizes, it is called a cast-off pattern (example: injuries by hammers) (James et al. 2005). In case of injury to the artery, the blood from the blood vessel flows like a fountain (upward to downward flow), a zigzag pattern will be observed until the pressure of the lungs reduces. If there was injury internally, expiration from the mouth/nose releases blood that creates a pattern very small to see (fine mist-like) (James et al. 2005; Peschel et al. 2011).

\section{Altered patterns}

Bloodstain patterns that indicate that a physical change had occurred can be said as altered patterns. This change can be due to physical activity, diffusion, dilution, or insects', which can misguide the investigators to consider them as drip patterns. In case if the body was dragged over pre-existing blood, it leaves a tangential 
path (James et al. 2005). Contact prints may also be recovered on clean surfaces at the crime scenes (bloody shoe prints, fingerprints, or the entire palm) that can help investigators in determining what might have occurred at the crime scene. This can help investigators to determine what object could have been at the crime scene (James et al. 2005; Peschel et al. 2011).

Void patterns on the other hands are formed when an object is placed between the blood source and projection area, it is likely to receive some of the stains, which consequently leads to an absence of the stains in an otherwise continuous bloodstain pattern, which can indicate that an object or person would have been a part of the pattern (like a missing object from the wall) that if recovered can help in completing the pattern (James et al. 2005; Peschel et al. 2011).

Insects that move over the blood can also create a unique pattern that can often confuse the investigators to what pattern it could be. When blood comes into contact with clothing and fabric it spreads via diffusion, often leaving an irregularly shaped pattern which is difficult to interpret, especially in that cases the surface could be collected and send for examination to forensic labs (James et al. 2005; Peschel et al. 2011).

Moreover, to reconstruct the events that caused bloodshed, the investigators use the direction and angle of the spatter to calculate the areas of convergence (it is the starting point of the bloodshed) and area of origin (point from where the blood immerged) to mark the location of the victim and perpetrator (James et al. 2005) (Fig. 1).

Different works have been carried in blood pattern analysis, a study showed that when determining area of origin from blood stains, larger drops which are elliptical should be given more consideration (de Bruin et al. 2011). In another study, the velocities of blood were considered with factors like air drag and gravity which was used to predict the back-spatter formation by carrying the experiment using a blood-soaked sponge (Comiskey et al. 2016).

Fluid dynamics was also given consideration in blood pattern analysis to understand how the blood behaves as a liquid when in air and the factors that are affecting the formation the blood drop (Attinger et al. 2013). Study of spines and satellite on basis of velocity has also depicted the formation of bloodstains (Attinger et al. 2013).

In a real-time setting, studying bloodstains and its patterns using real blood can be a tedious task, as it requires a large amount of blood. Moreover, in order to carry out such a study, it will require ethical clearance as well as financial support. Using Awlata dye for studying bloodstains can solve these problems because of its easy availability, low cost, and it can be made under

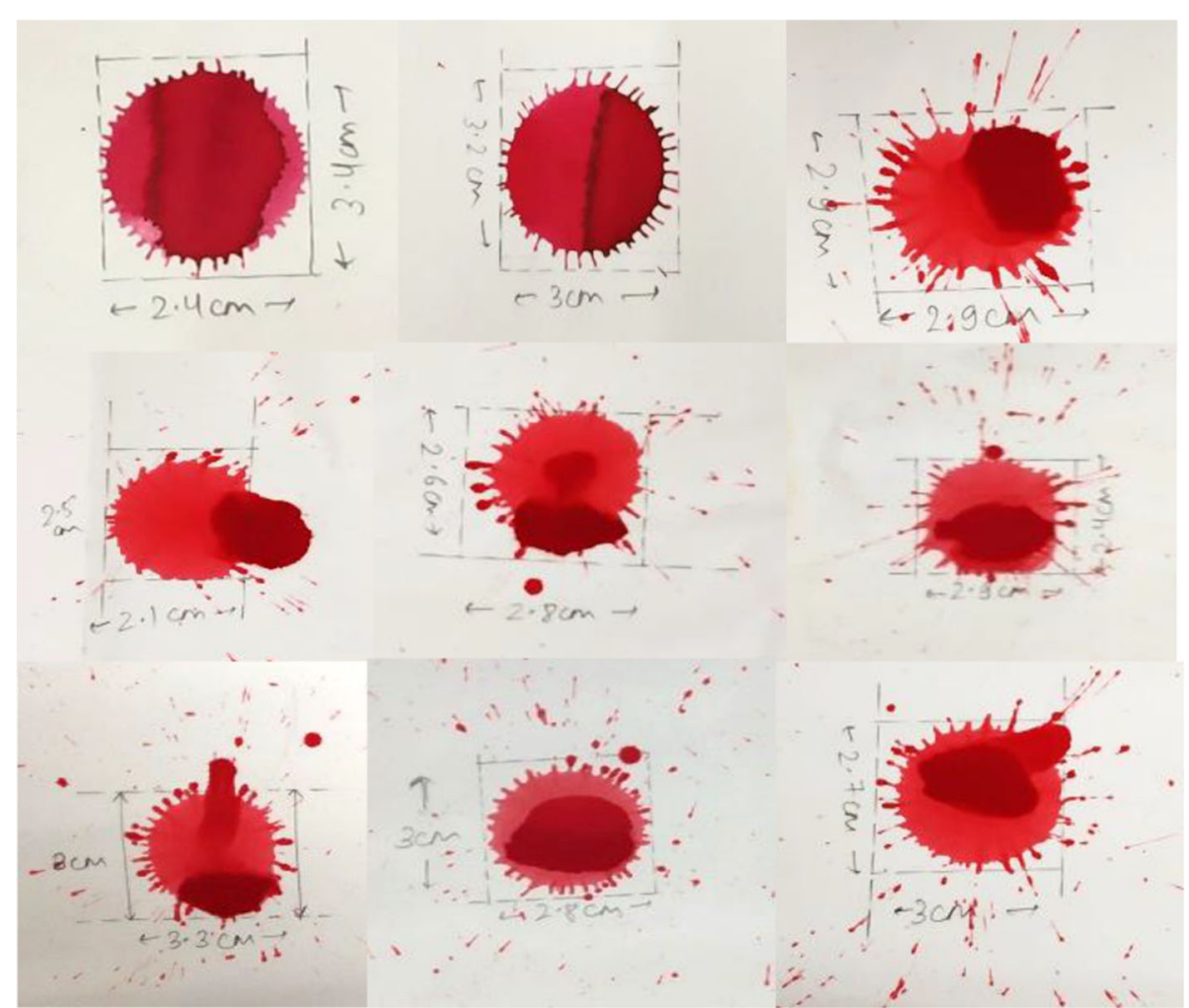

Fig. 1 Fake blood stains that were made using Awlata dye 
laboratory conditions. Thus, investigators and scientists can use this for experimental purposes and to carry future studies.

\section{Methods}

\section{Article selection criteria for review}

The initial criteria for selecting literature were based on searching different keywords on Google searching engine for blood, blood pattern analysis, and blood pattern analysis in forensic science. Then, after screening of articles based on the title and abstract of papers, papers were sorted. Articles and relevant internet sources that matched the relevant criteria of the review were also selected.

\section{Article eligibility criteria for review}

Eligibility of articles was finalized by analyzing whether the papers were discussing about BPA and its related methodology or not.

\section{Materials}

On basis of analyzing existing literature, it was decided that a study needs to be conducted by creating fake bloodstains using Awlata, so as to understand the formation of stains if the angle is kept fixed and the height is varied (Buck et al. 2011; Attinger et al. 2013). An Indian dye (Awlata/Alta) was used to make fake blood stains to depict similar patterns as that of blood. Awlata (Alta) is a traditional Indian red dye used by women in the festive season and is applied to hands and feet. For the experiment Awlata dye, a Pasteur pipette and white chart papers were used. The experiment was carried within the university premises in the university laboratory.

\section{Preparation/composition of Awlata}

In cultural practices, Awlata dye was made from Betel leaves which is a vine from the family Piperaceae. Awlata is also made from the extract of lac that is a red dye obtained from the scale of an insect Laccifer Lacca. Nowadays, Awlata can be made chemically by using Vermillion (red powder) with water to make a liquid.

\section{Source of Awlata for the experiment}

For this experiment, a ready-made Awlata dye (Pari) was bought from the local market which had its composition defined and came packed in a $50-\mathrm{ml}$ bottle. The reason for taking Awlata for experiment, was Awlata dries within a few minutes and its life span is about 1-2 months, after which it starts to fade. But if it is preserved and stored properly, it can stay intact for long durations.

\section{Formation of fake bloodstains}

In this experiment, we conducted different height variations to create fake bloodstains using Awlata dye (Buck et al. 2011; Attinger et al. 2013). The experiment aimed to study the shape (morphology) of these fake bloodstains at different heights $(3,4,5,6$, and 7 feet) so that an approximate estimation of actual blood stain formation can be studied (Attinger et al. 2013). A Pasteur pipette was used for this experiment and about $0.5 \mathrm{ml}$ of Awlata dye was taken for making a single fake blood stain.

The amount of Awlata dye that was used to make a single fake blood stain was ascertained using the indications labeled on the Pasteur pipette. Ninety-degree angle was maintained and the Awlata dye was dropped from different heights and observations were made. For each height, two stains were made and labeled drop 1 and drop 2; this was done to compare the observation and confirm the findings.

\section{Results}

After Awlata dye was dropped from different heights to create fake blood stains, it was observed that as the height was increased, the distance of satellite stains emerging from the fake blood stains was also increasing. It can be observed that fake blood stain created from three feet has many numbers of spines and less satellite stains and they are very close to the parent stain. Similarly, as the height was increased, the numbers of spines were reduced and the number of satellite stains was increased.

The experimental observation noted was that as the height was increased, the force of gravity acting on the Awlata dye was also increasing, and hence when the Awlata dye was dropped from a height to create fake blood stains, the impact of the dye on the surface due to gravitational forces, inertial forces, and viscous forces (Attinger et al. 2013) could be the possible cause of formation of such stains. To ascertain the formation of these fake blood stains, we retook the height experiment and the observations were very similar to that of the first drop (see Table 1).

\section{Discussions}

After the experiment carried with Awlata dye, it was observed that height was directly proportional to the number of satellite stains (stains that are small droplets moving away from the parent stain, they are partially/ not attached to the parent stain), i.e., more distant the satellite stains from the parent drop, more will be the height. Whereas relation of spines (these are small projections coming out from the parent stain, they remain attached to the parent stain) and height was inverse in nature, i.e., when the height was increased the number of spines reduced.

Though Awlata was used to study the formation of fake blood stains, care must be taken that this dye should be kept away from contact with moisture/water as repeated moisture/water tends to fade the dye and 
Table 1 Experiment depicting effect of height on formation of blood drop

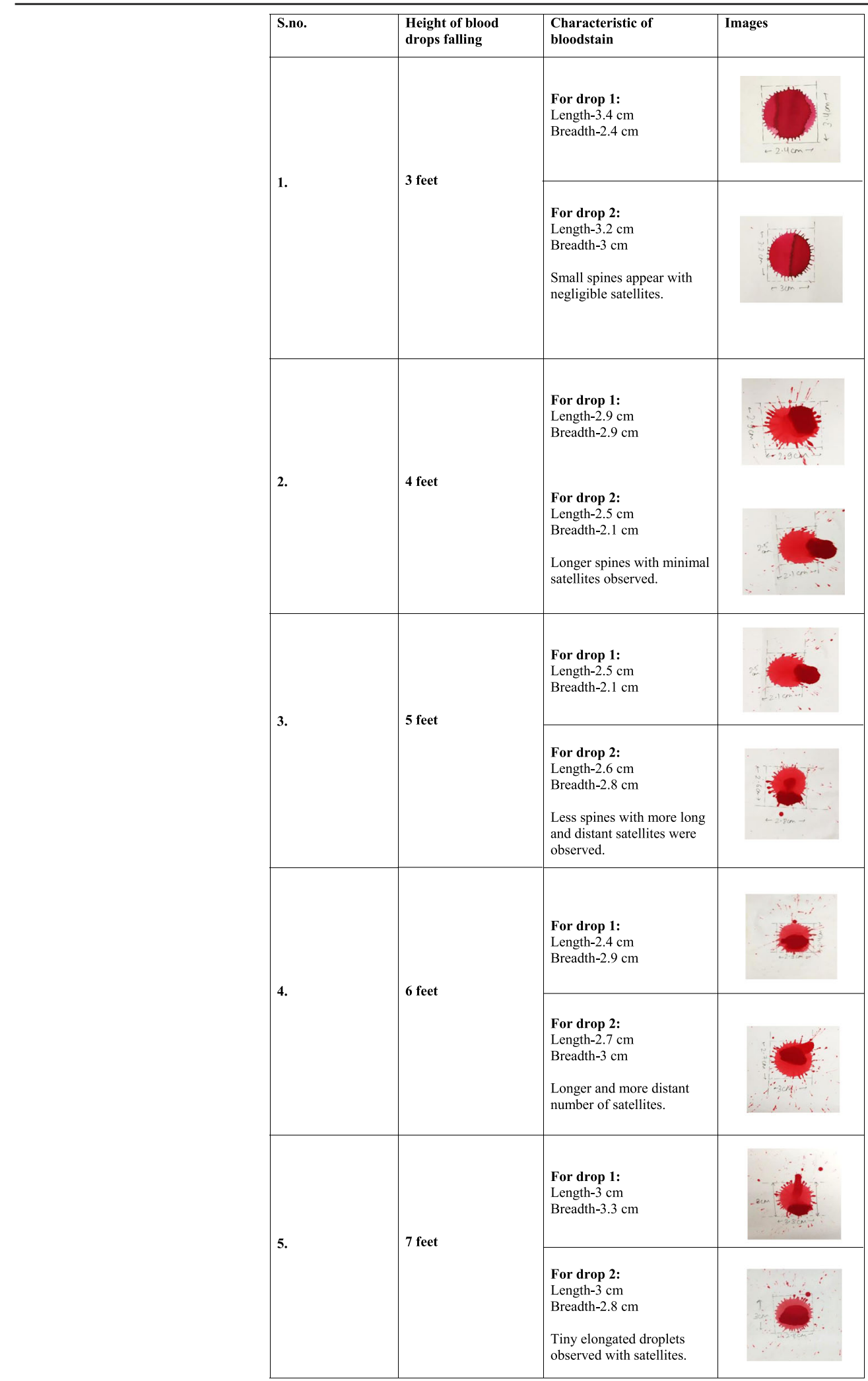


also wash it off. So, if Awlata dye is used for future studies, the observations made from this dye should be properly stored and preserved. This will help ensure the integrity of experimental findings is not altered.

Factors like size, age, and health of the individual should also be given consideration while studying the blood stain formation. Moreover, surface tension also plays an important role in the formation of bloodstains (Larkin et al. 2012). Surface tension is also varied if there is some chemical or other chemical present in the blood (Raymond et al. 1996). The surface roughness, permeability, and porosity also effect the formation of bloodstain formation. So, these factors are also needed to be given consideration when studying bloodstains (Bear 1975).

Study of fake bloodstains using Awlata dye highlights these potential aspects and on basis of existing literary works carried by other scientists a more definite version of BPA can be worked upon. Study of fluid dynamics should also be given consideration while studying bloodstain formation (Attinger et al. 2013). To open new gateways of research in BPA and support investigative observations, the findings depicted in this paper can be used as a source to validate actual blood stains and also carry out future studies. Domains like how angle variation with respect to height effects formation of blood stains can be explored on the basis of these findings. This finding can help to understand the formation of blood stains for future research and development.

The future of BPA is promising and more research needs to be done to improve BPA. A more precise method of blood interpretations should be created to make investigations more accurate, so that crime scene reconstruction can be carried out efficiently. The study conducted using Awlata dye can be a contributor to the existing literature on BPA. This paper is a review work which can be utilized by students, scientists, or experts as a reference for carrying out future studies or to enhance their knowledge. Blood pattern analysis is indeed a useful tool in forensic science which can help in crime scene reconstruction and if BPA is coupled with DNA analysis and other investigative findings, more conclusive and thorough details of the sequence of events can be obtained from blood evidence.

\section{Limitations of Awlata dye}

The composition of Awlata dye (Alta) and blood vary; hence, Awlata dye (Alta) cannot be considered as blood. Awlata was used to create fake bloodstains which can give an approximate idea towards BPA and resemblance somewhat similar to actual blood stains. The actual scenario at the crime scene that led to the formation of blood stains and that made by Awlata dye has scope for human errors too as studying blood stains in real and that in experimental conditions differ.

\section{Awlata dye use in the forensic scenario}

Studying BPA is a very skillful task, and at the crime scene when real blood is concerned, the scenarios are simultaneous and unpredictable. To carry studies to understand bloodstains is not always possible; it requires a large amount of blood which is subjected to ethical clearance. Awlata dye can be an emerging substitute to this problem, as it is cost-effective, readily available, and can also be made in the lab. Awlata dye can be used to create experimental conditions to study different forensic scenarios. Fake blood created with Awlata dyes can be used to make simulated crime scenes from forensic and investigative findings to derive case supportive conclusions.

\section{Conclusion}

From the experiment done using Awlata dye (Alta), it can be concluded that blood stains can help experts estimate the approximate height of the assailant. The formation of the bloodstain can correspond to the height it originated from, thus being a vital source of information. A relation of formation of blood stains with change in varying height was established in accordance with interpretation of spines and satellite stains. Though Awlata is not similar to blood, it can be used to carry out experimental studies to explore more about BPA. Existing studies on BPA depict that blood patterns are very useful source of information and it can help investigators to examine the crime scene precisely.

\section{Abbreviations}

BPA: Blood pattern analysis; DNA: Deoxyribonucleic acid; UV: Ultra violet

\section{Acknowledgements}

Not applicable

\section{Authors' contributions}

PS and NG worked on researching relevant data and writing of this review paper. RR was our guide and mentor who constantly guided us and helped formulate the different sections of the review and did the final check. We clarify that all authors have read and approved the final manuscript.

\section{Funding}

Not applicable

Availability of data and materials Not applicable

\section{Declarations}

Ethics approval and consent to participate Not applicable

\section{Consent for publication \\ Not applicable}

Competing interests

The authors declare that they have no competing interests. 


\section{Author details}

'School of Forensic Science, National Forensic Sciences University, Gandhinagar, Gujarat, India. ${ }^{2}$ Department of Chemistry, Biochemistry and Forensic Sciences, Amity University, Manesar, Haryana, India.

Received: 28 September 2020 Accepted: 9 May 2021

Published online: 21 May 2021

\section{References}

Attinger D, Moore C, Donaldson A, Jafari A, Stone H (2013) Fluid dynamics topics in bloodstain pattern analysis: Comparative review and research opportunities. Forensic Sci Int 231(1-3):375-396. https://doi.org/10.1016/j. forsciint.2013.04.018

Barni F, Lewis S, Berti A, Miskelly G, Lago G (2007) Forensic application of the luminol reaction as a presumptive test for latent blood detection. Talanta 72(3):896-913. https://doi.org/10.1016/j.talanta.2006.12.045

Bear J (1975) Dynamics of fluids in porous media. Soil Sci 120(2):162-163. https:// doi.org/10.1097/00010694-197508000-00022

Bevel T, Gardner R (2012) Bloodstain pattern analysis with an introduction to crime scene reconstruction, 3rd edn. Taylor and Francis, Hoboken

Brodbeck S (2012) Introduction to bloodstain pattern analysis. SIAK J J Police Sci Pract 2:51-57 Avaialble via DIALOG. https://www.bmi.gv.at/104/Wissenschaft_ und_Forschung/SIAK-Journal/internationalEdition/files/Brodbeck_IE_2012.pdf

Buck U, Kneubuehl B, Näther S, Albertini N, Schmidt L, Thali M (2011) 3D bloodstain pattern analysis: Ballistic reconstruction of the trajectories of blood drops and determination of the centres of origin of the bloodstains. Forensic Sci Int 206(1-3):22-28. https://doi.org/10.1016/j.forsciint.2010.06.010

Comiskey P, Yarin A, Kim S, Attinger D (2016) Prediction of blood back spatter from a gunshot in bloodstain pattern analysis. Phys Rev Fluids. 1(4). https:// doi.org/10.1103/physrevfluids.1.043201

de Bruin K, Stoel R, Limborgh J (2011) Improving the point of origin determination in bloodstain pattern analysis. J Forensic Sci 56(6):1476-1482. https://doi.org/10.1111/j.1556-4029.2011.01841.x

James S, Kish P, Sutton T (2005) Principles of bloodstain analysis. CRC, Boca Raton, Fla. https://doi.org/10.1201/9781420039467

Karger B, Rand S, Fracasso T, Pfeiffer H (2008) Bloodstain pattern analysis-casework experience. Forensic Sci Int 181(1-3):15-20. https://doi. org/10.1016/j.forsciint.2008.07.010

Kunz S, Brandtner H, Meyer H (2013) Unusual blood spatter patterns on the firearm and hand: a backspatter analysis to reconstruct the position and orientation of a firearm. Forensic Sci Int 228(1-3):e54-e57. https://doi.org/10.1 016/j.forsciint.2013.02.012

Kunz S, Brandtner H, Meyer H (2015) Characteristics of backspatter on the firearm and shooting hand-an experimental analysis of close-range gunshots. J Forensic Sci 60(1):166-170. https://doi.org/10.1111/1556-4029.12572

Larkin B, El-Sayed M, Brownson D, Banks C (2012) Crime scene investigation III: exploring the effects of drugs of abuse and neurotransmitters on bloodstain pattern analysis. Anal Methods 4(3):721. https://doi.org/10.1039/c2ay05762j

Peschel O, Kunz S, Rothschild M, Mützel E (2011) Blood stain pattern analysis. Forensic Sci Med Pathol 7(3):257-270. https://doi.org/10.1007/s12024-010-9198-1

Quickenden T, Creamer J (2001) A study of common interferences with the forensic luminol test for blood. Luminescence 16(4):295-298. https://doi. org/10.1002/bio.657

Raymond M, Smith E, Liesegang J (1996) The physical properties of bloodforensic considerations. Sci Justice 36(3):153-160. https://doi.org/10.1016/s13 55-0306(96)72590-x

Saferstein R, Hall A (2020) Forensic science handbook. CRC Press, Taylor \& Francis Group, Milton. https://doi.org/10.4324/9781315119939

Swgstain S (2009) Scientific Working Group on bloodstain pattern analysis: recommended terminology. Forensic Sci Commun 11(2):14-17 Available via DIALOG. http://theiai.org/docs/SWGSTAIN_Terminology.pdf

\section{Publisher's Note}

Springer Nature remains neutral with regard to jurisdictional claims in published maps and institutional affiliations.

\section{Submit your manuscript to a SpringerOpen ${ }^{\circ}$ journal and benefit from:}

- Convenient online submission

- Rigorous peer review

- Open access: articles freely available online

- High visibility within the field

- Retaining the copyright to your article

Submit your next manuscript at $\boldsymbol{\nabla}$ springeropen.com 